\title{
Analysis of the functional diversity of the microbial communities in a paper-mill water system
}

\author{
Thea van der Merwe ${ }^{1 *}$, Francois Wolfaardt ${ }^{1,2}$ and Karl-Heinz Riedel ${ }^{3}$ \\ ${ }^{1}$ Department of Microbiology and Biochemistry, PO Box 339, University of the Free State, Bloemfontein 9300, South Africa \\ ${ }^{2}$ SAPPI Forest Products, Technology Centre, PO Box 3252, Springs 1560, South Africa \\ ${ }^{3}$ School for Environmental Sciences and Development: Microbiology, Potchefstroom University for Christian Higher Education, \\ Potchefstroom 2520, South Africa
}

\begin{abstract}
Most microbiological studies of wastewater treatment systems rely on methods that are dependent on culturing. However, due to the limitation of conventional microbiological methods, alternative assays, such as the analysis of the functional diversity of the microbial communities using Biolog substrate utilisation profiles, have been proposed. The Biolog method was thus evaluated in a paper-mill water system. The influence of the production of various paper grades, biocide combinations and monthly maintenance shut-downs on the functional diversity of the microbial communities were determined using the Biolog technique. The communities in the planktonic as well as the sessile phases were analysed approximately every second week for a period of one year. The average well colour development (AWCD) technique was used to transform the data obtained from the Biolog plates prior to multivariate statistical analysis. Our results indicated that different microbial communities developed during the production of the different paper grades. A difference in substrate utilisation was evident after a single day of production of fluting or linerboard in the planktonic communities, while differences in the sessile communities only became significant after an extended period of production. The effect of the application of different biocides was more distinct in the planktonic than in the sessile populations. No clear trends concerning the influence of maintenance shut-downs and cleaning of machines could be observed in the sessile phase, while a shift in the microbial community could be observed in the planktonic phase. Biodiversity indices indicated that a high functional diversity existed in both the planktonic and sessile phases. It was concluded that the substrate utilisation profiles obtained with Biolog plates were a sensitive measure, which enabled the detection of shifts in the microbial community function and possibly structure within the same water system.
\end{abstract}

\section{Introduction}

Most microbiological studies of wastewater treatment systems rely on cultivation, which quantifies a limited percentage of the microbial populations present (Schneider et al., 1998). Incorrect conclusions concerning the function and structure of the microbial community in situ are consequently made. Due to the limitation of conventional microbiological methods, numerous alternative techniques that circumvent these limitations have been developed to characterise microbial communities in situ.

One such a technique is the analysis of the functional diversity of the microbial community (Buyer and Drinkwater, 1997; Garland and Mills, 1991; Zak et al., 1994). Zak et al. (1994) defined the functional diversity of a microbial community as the numbers, types, activities and rates at which a suite of substrates is utilised. Garland and Mills (1991) proposed that substrate utilisation, using commercially available Biolog plates, could be used to characterise the functional differences among microbial communities. The application of the substrate utilisation assay has been studied extensively in various environments such as soil, water (Garland and Mills, 1991) and wastewater systems (Victorio et al., 1996).

Due to the success of the application of the substrate utilisation approach, it was decided to test this system in an industrial water system. The aim of this study was, therefore, to monitor the functional diversity of the microbial community in the water system of the Sappi Cape Kraft paper-mill, South Africa, over a period of one year. The influence of the production of various paper

* To whom all correspondence should be addressed.

急051-4013332; fax 051-4443219; e-mail: vdmerwd@sci.uovs.ac.za Received 28 June 2002; accepted in revised form 16 October 2002. grades, types of biocide used and monthly maintenance shutdowns on the substrate utilisation profiles for both the planktonic and sessile microbial communities was studied during this period.

The Sappi Cape Kraft paper-mill, Cape Town, South Africa manufactures both fluting and linerboard from recycled fibre and reuses a large percentage of the process water. Fluting is produced at $\mathrm{pH}$ values between 6.5 and $\mathrm{pH} 7.5$ while linerboard is produced at $\mathrm{pH}$ values between 4.5 and $\mathrm{pH}$ 5.5. Aluminium sulphate and rosin size are added during the production of linerboard to improve the printing quality and to make storage under conditions of high humidity possible. Fluting is produced without any additives.

Different combinations of biocide formulations were added to the clarifier to control microbial fouling. The first combination consisted of a formulation with organosulphur (sulphone) and quaternary ammonium compounds and a formulation of organosulphur (carbamate). The organosulphur (sulphone) and quaternary ammonium compounds are effective against bacteria and fungi (Boiven, 1995; McCoy, 1980). It has also been reported that organosulphur (carbamate) is very effective against sulphate reducing bacteria (Boiven, 1995). The second combination consisted of a formulation with quaternary ammonium compounds and organotin that is more effective against fungi than bacteria (Drew Industrial Division, 1994).

\section{Materials and methods}

\section{Sample preparation and incubation}

Duplicate planktonic and sessile samples $(\sim 50 \mathrm{~m} \ell)$ were collected fortnightly from the Cape Kraft mill for a period of one year. Approximately $5 \mathrm{~m} \ell$ of biofilm or $10 \mathrm{~m} \ell$ of the planktonic phase 


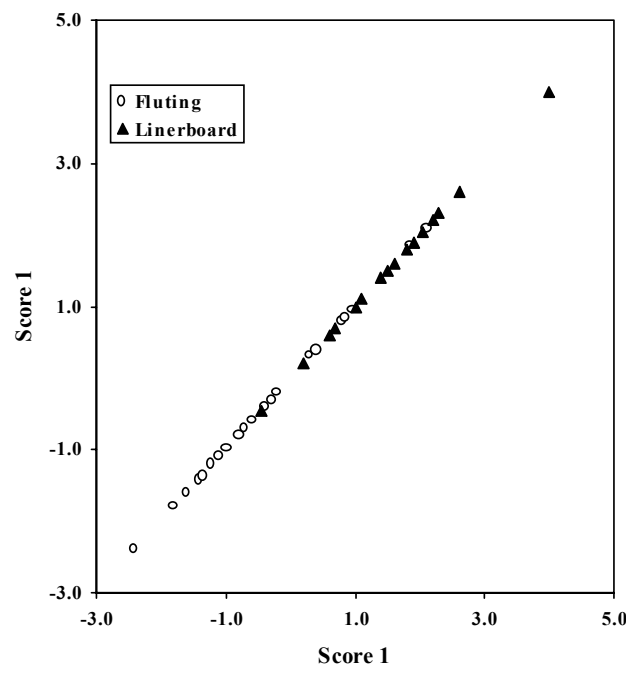

Figure 1

Ordination plot of the canonicalvariates scores obtained for the planktonic microbial community after $1 d$ of production of each paper grade

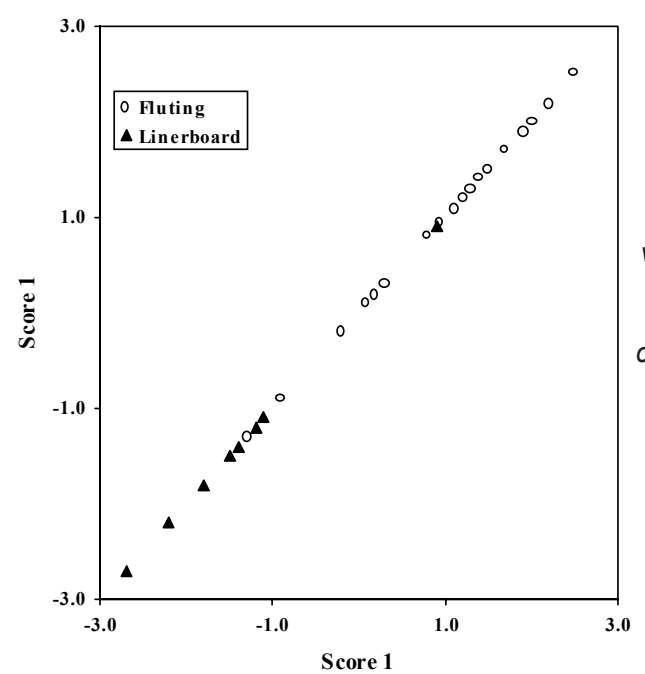

Figure 3

Ordination plot of the canonicalvariates scores for the sessile microbial community after $5 d$ of production of each paper grade

from the different samples was added to $30 \mathrm{~m} \ell$ of phosphate buffer (Guckert et al., 1996), homogenised (Heidolph Diax 600, Germany) and centrifuged ( $500 \mathrm{G}$ for $1 \mathrm{~min}$ ) to remove any fibres. The supernatant was standardised to a turbidity of 0.25 to 0.35 Absorbance units $(420 \mathrm{~nm})$ using the phosphate buffer. Gramnegative (GN) Biolog plates (Biolog Inc., Hayward, USA) containing 95 different carbon sources were used during this study and inoculated with $150 \mu$ of the standardised sample. Assimilation of the carbon sources was reflected by the reduction of tetrazolium violet. Duplicate Biolog plates were evaluated for absorbance changes at $590 \mathrm{~nm}$ using a Labsystems iEMS microtiter plate analyser (Labsystems, Helsinki, Finland). Following an initial (time 0) reading, the Biolog plates were incubated at $25^{\circ} \mathrm{C}$ in the dark and the reduction of tetrazolium violet was measured after 12 , 24, 36, 48 and $64 \mathrm{~h}$.

The data obtained from the Biolog microplates were standardised using the average well colour development (AWCD) technique (Garland, 1996). An AWCD value of 0,3 to 0,5 Absorbance units was used as the reference point for statistical analysis of the data (Heuer and Smalla, 1997).

\section{Data analysis}

Data were analysed using the NCSS 97 software (Statistical Solutions, Ireland). A carbon source was assumed to be important for the discrimination between profiles when the standardised OD was significantly different $(p<0.05)$ between samples and its
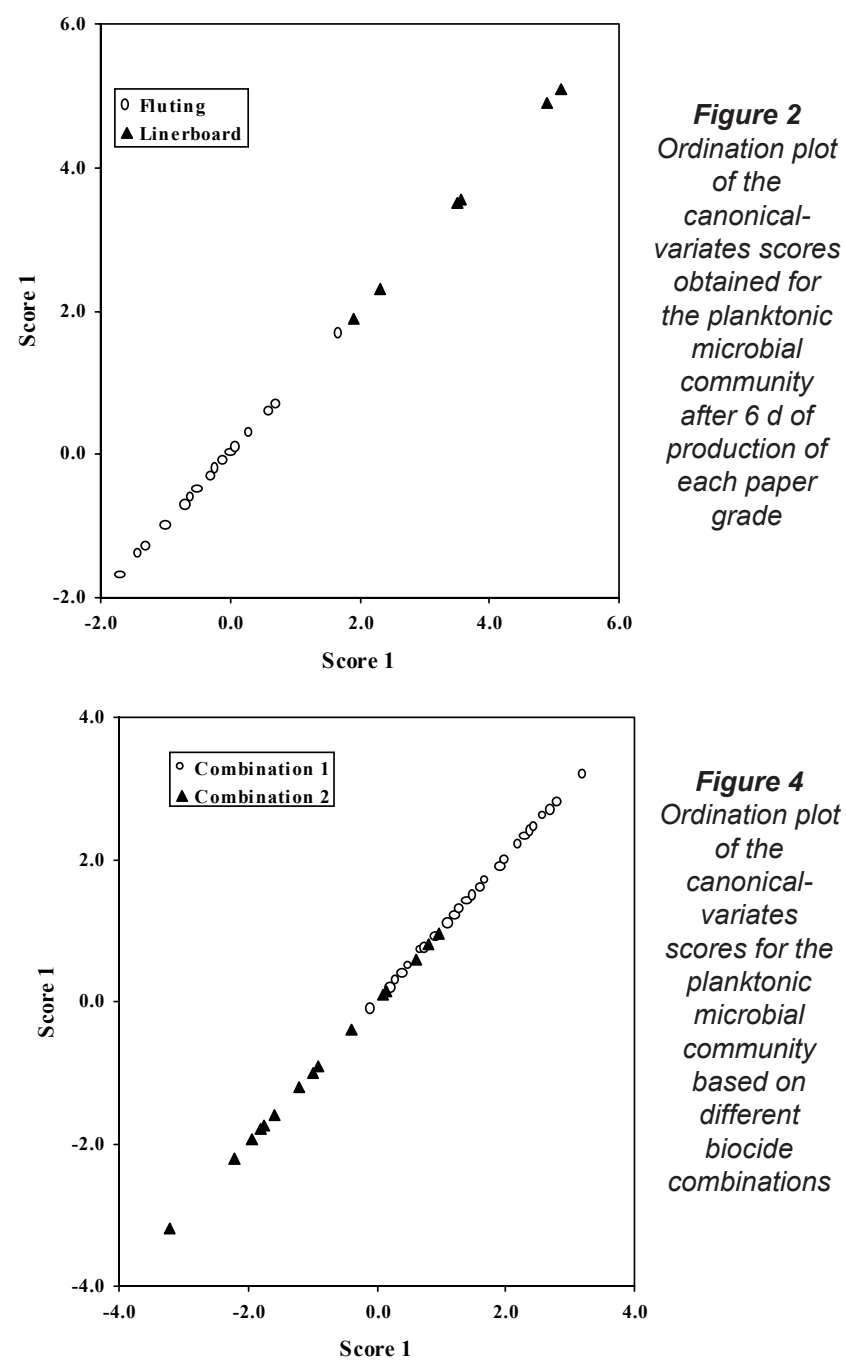

weighted sum of factor-loadings was higher than the median, suggesting a significant contribution to the multivariate statistical difference (Heuer and Smalla, 1997). The Wilks' $\lambda$ was used to test the significance of the discriminant function, where values close to one implied a low predictability and values close to zero implied a high predictability. The F-ratio was used to test the significance of the Wilks' $\lambda$ (Hintze, 1997).

\section{Functional diversity}

The influence of different biocide combinations on the functional diversity in water and biofilm samples was determined by comparison of the diversity indices for sixteen consecutive samples over a sixteen week period. The first eight samples were collected when the first biocide combination was applied and the last eight when the second biocide combination was applied to the process water.

The functional diversity of the analysed microbial communities was quantified using a substrate diversity index or modified Shannon index (Magurran, 1988; Zak et al., 1994):

$$
H^{\prime}=-\Sigma\left[p_{\mathrm{i}} \ln \left(p_{\mathrm{i}}\right)\right]
$$

where:

$p_{\mathrm{i}}$ is the ratio of the corrected $\mathrm{OD}_{590}$ value for a carbon source to the sum of the corrected $\mathrm{OD}_{590}$ values for all substrates. 


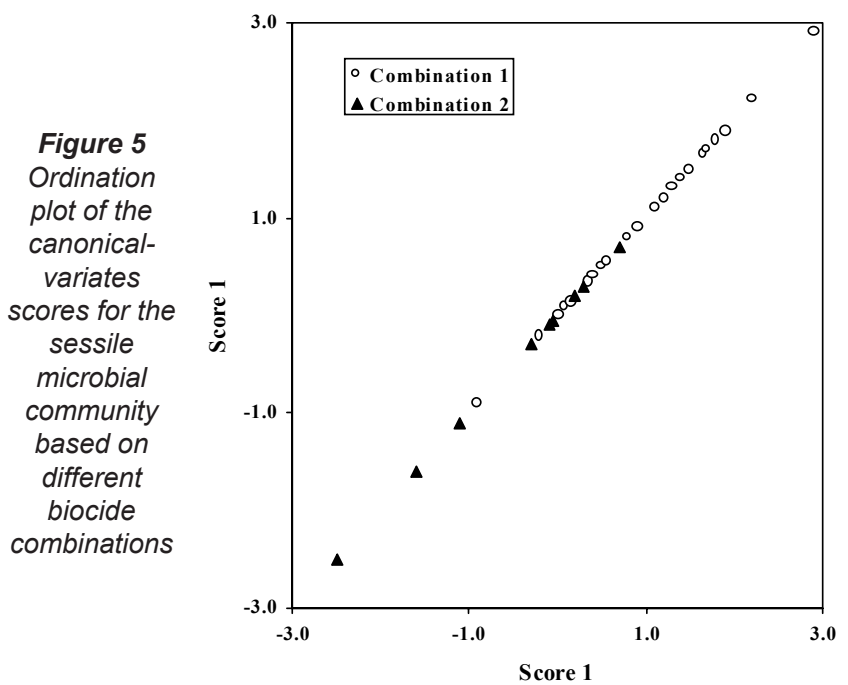

The substrate equitability or evenness $(J)$ was calculated by rescaling the substrate diversity index (Magurran, 1988; Zak et al., 1994):

$$
J=H^{\prime} / H_{\max }^{\prime}
$$

where:

$H^{\prime}{ }_{\max }$ is the maximal substrate diversity index for all the samples.

\section{Results and discussion}

The substrate utilisation profiles of the planktonic microbial community when fluting was produced differed significantly (Wilks' $\lambda=0.52 ; \mathrm{F}=12.4$ ) from the community when linerboard was produced (Fig. 1). These differences were apparent within a single day after the production process was changed, although some overlapping between the substrate utilisation profiles of these communities was apparent (Fig. 1). When the specific production process continued, the differences in the substrate utilisation of the microbial communities in the planktonic phase became more pronounced. Less overlapping in the overall substrate utilisation profiles of the microbial communities were apparent (Fig. 2) when compared to that observed after $1 \mathrm{~d}$ of production (Fig. 1). The decrease in the Wilks' $\lambda$ value (to 0.25 ) after $6 \mathrm{~d}$ of production showed a significant improvement in the predictability of the model, since values close to zero are indicative of a very accurate model (Hintze, 1997). The shifts in the substrate utilisation profiles of the microbial communities could possibly be ascribed to the differences in $\mathrm{pH}$ values of the process water during the production of fluting and linerboard, as well as the addition of aluminium sulphate and rosin size during the production of linerboard. At higher $\mathrm{pH}$ values, bacteria can become more abundant (Robertson, 1993) and populations with the ability to assimilate the additives could develop.

The substrate utilisation profiles of the microbial communities in the sessile phase differed significantly (Wilks' $\lambda=0.46 ; \mathrm{F}=15.2$ ) only after $5 \mathrm{~d}$ of production of a specific paper grade (Fig. 3). The substrate utilisation profiles of the microbial communities in the sessile phase remained relatively constant and did not respond immediately to the change in the production of the different paper grades. This was in contrast to the functionality of the planktonic phase of the paper-mill water system, where a shift in the microbial community could be detected immediately. These results confirmed the protective effect of biofilms where the micro-organisms are not as exposed to environmental stress (Johnsrud, 1997).

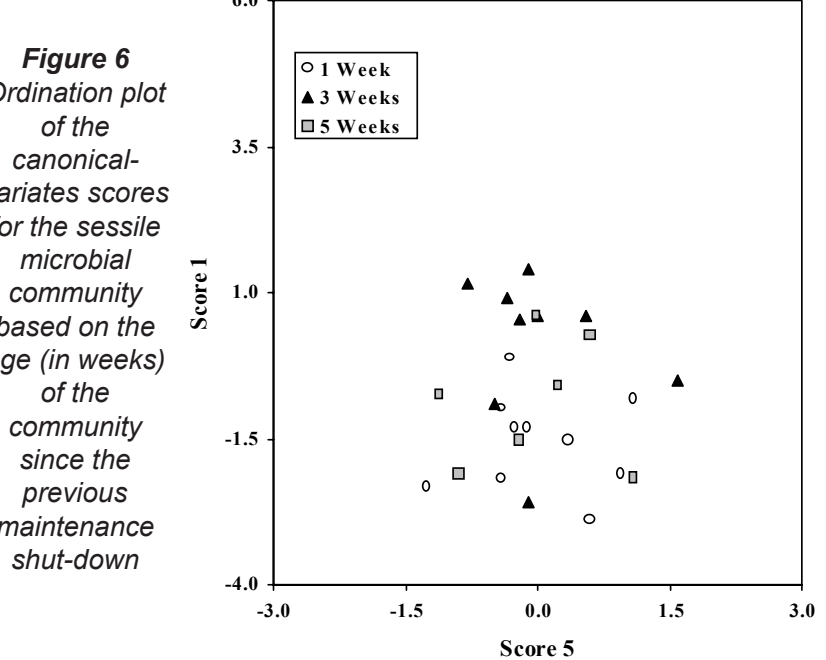

Figure 7

Ordination plot of the

canonical-

variates scores

for the

planktonic

microbial

community

based on the

age (in weeks)

of the

community

since the

previous

maintenance

shut-down

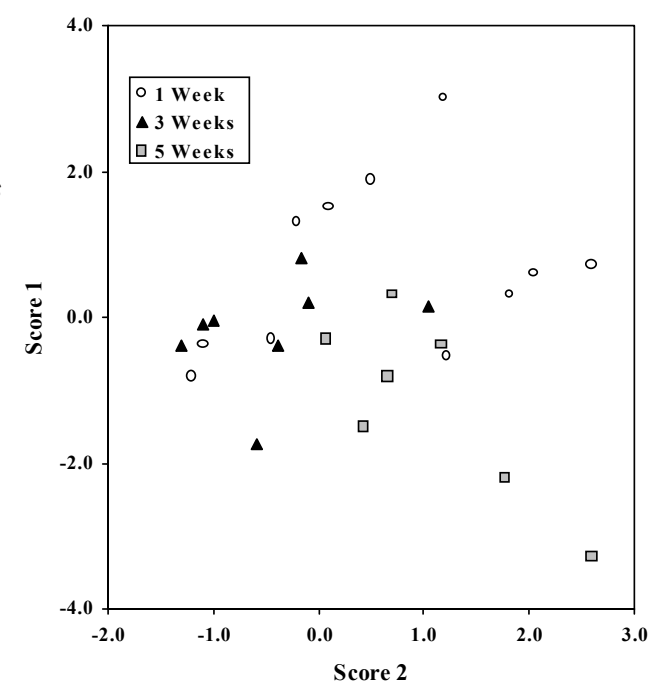

TABLE 1

Average substrate diversity $\left(H^{\prime}\right)$ and substrate equitability $(J)$ values obtained for two biocide combinations over a sixteen week period

\begin{tabular}{|l|l|c|c|}
\hline \multicolumn{2}{|c|}{} & \multicolumn{2}{c|}{$\begin{array}{c}\text { Biocide } \\
\text { combination }\end{array}$} \\
\cline { 3 - 4 } \multicolumn{2}{|c|}{} & A & B \\
\hline \multirow{2}{*}{ Planktonic phase } & Shannon index (H') & 3.864 & 4.049 \\
& Evenness (J) & 0.849 & 0.889 \\
\hline \multirow{2}{*}{ Sessile phase } & Shannon index (H') & 4.185 & 4.099 \\
& Evenness (J) & 0.892 & 0.901 \\
\hline
\end{tabular}

The substrate utilisation profiles of the microbial communities in the planktonic and sessile samples differed significantly (Wilks' $\lambda=0.40 ; \mathrm{F}=21.2$ and Wilks' $\lambda=0.61 ; \mathrm{F}=28.0$ ) when the two different biocide combinations were applied to the water system (Figs. 4 and 5). Indications are that the different biocides resulted in microbial communities with differences in their nutritional preferences. Comparison of substrate diversity $\left(H^{\prime}\right)$ and evenness $(J)$ indices indicated that the diversity in the microbial populations did not differ significantly between biocide combinations (Table 1). Toxic environments are characterised by reduced biodiversity 
and increased dominance (Magurran, 1988). Despite the fact that the biocide combinations resulted in different microbial populations in both the planktonic and sessile phases, there was no significant decrease in the biodiversity or evenness, therefore, both biocide combinations appear to be ineffective.

Age was defined as the time (in weeks) that elapsed after a maintenance shut-down. The substrate utilisation profiles of the sessile populations did not change significantly as they aged (Fig. 6). This stability could possibly be ascribed to inefficient cleaning during the maintenance shut-downs at the mill. In contrast, the substrate utilisation of the microbial communities in the planktonic phase changed with age after cleaning during maintenance shutdowns, possibly due to clean water entering the system (Fig. 7).

High substrate diversity indices $\left(H^{\prime}\right)$ were measured in all the planktonic and sessile samples. These high values are a reflection of the fact that most of the carbon sources were utilised, contributing to the very high Shannon indices. The evenness indices $(J)$ obtained for the planktonic and sessile samples did not show any trends or significant differences between samples. Magguran (1998) stated that a high equitability is an indication of a large biodiversity. The substrate diversity remained relatively stable in the sessile $\left(3.58<H^{\prime}<4.43\right)$ and planktonic $\left(3.36<H^{\prime}<4.40\right)$ samples despite the continuous change in the various production parameters (paper grade and biocide dosage). No correlation between these parameters and the different diversity indices could, however, be found.

\section{Conclusions}

On the basis of substrate utilisation profiles, it was observed that different microbial communities developed in the process water system when linerboard and fluting were produced. The shifts in the substrate utilisation were probably caused by the changes in $\mathrm{pH}$ and chemical additives during the various production processes. The results obtained during the present study were similar to results obtained by Kaiser et al. (1998), who reported that substrate utilisation was a sensitive method to detect differences in the metabolic responses of mixed microbial communities. The planktonic microbial communities in the water system were much more sensitive to changes in the external environment, with the shifts in the substrate utilisation profiles of the microbial communities being much more pronounced and rapid when compared to the sessile microbial communities. This could possibly be due to the protective effect of biofilms and the fact that attached micro-organisms may be better adapted to their environment than those occurring in the planktonic phase (Robertson, 1994).

The two different biocide combinations selected for communities with differences in their nutritional preferences, although slight overlapping was evident. The overlapping of the microbial communities may be the result of the selection of specific microbial populations by the biocides.

The results also suggested that different biocides should be used when different paper grades are manufactured. The implementation of a microbial control strategy at the plant will have to take the variation in the production process into consideration and biocides will have to be selected for specific production processes. Biocide dosage into the process water system would have an immediate effect on the micro-organisms present in the planktonic phase, whilst the response in the sessile phase would be delayed.

It was also apparent that age did not have a significant influence on the substrate utilisation profiles of the microbial communities in the sessile phase, possibly due to inefficient cleaning during maintenance. In contrast, differences in the substrate utilisation profiles of the microbial communities within the planktonic phase were observed. The high substrate equitability values indicated that the microbial communities in the planktonic and sessile phase utilised the carbon sources in the same proportion. The high substrate diversity values also implied a high functional diversity.

In conclusion, it was evident that carbon source utilisation profiles provide an alternative technique to conventional culturing for the characterisation of microbial communities. This technique was also sensitive enough to detect shifts in the microbial communities due to changes in the operational parameters of the paper-mill. This approach could, therefore, be of significant value during the selection and implementation of microbial control programmes in this and other industrial water systems.

\section{Acknowledgements}

The authors are grateful for the assistance of Fransa Burger from the University of the Free State, Ken Payten from South African Paper Chemicals, Amanda Horne from Sappi Cape Kraft and the financial support from the Department of Trade and Industry.

\section{References}

BOIVEN J (1995) Oil industry biocides. Mat. Perf. 34 65-68.

BUYER JS and DRINKWATER LE (1997) Comparison of substrate utilisation assay and fatty acid analysis of soil microbial communities. J. Microbiol. Methods 30 3-11.

DREW INDUSTRIAL DIVISION (1994) Principles of Industrial Water Treatment. NJ Ashland Chemical Co, Boonton.

GARLAND JL and MILLS AL (1991) Classification and characterisation of heterotrophic microbial communities on the basis of patterns of community-level sole-carbon-source utilisation. Appl. Environ. Microbiol. 57 (8) 2351-2359.

GARLAND JL (1996) Analytical approaches to the characterisation of samples of microbial communities using patterns of potential C source utilisation. Soil Biol. Biochem. 28 213-221.

GUCKERT JB, CARR GJ, JOHNSON TD, HAMM BG, DAVIDSON DH and KUMAGAI Y (1996) Community analysis by Biolog: Curve integration for statistical analysis of activated sludge microbial habitats. J. Microbiol. Methods 27 183-197.

HEUER H and SMALLA K (1997) Evaluation of community level catabolic profiling using BIOLOG GN microplates to study microbial community changes in potato phylloshere. J. Microbiol. Methods 30 113-120.

HINTZE J (1997) NCSS Users Manual. Statistical Solutions, Ireland.

JOHNSRUD SC (1997) Biotechnology for solving slime problems in the paper and pulp industry. Adv. Biochem. Eng. \& Biotech. 57 312-328.

KAISER SK, GUCKERT JB and GLEDHILL DW (1998) Comparison of activated sludge microbial communities using Biolog ${ }^{\mathrm{TM}}$ microplates. Water Sci. Technol. 37 (4-5) 57-63.

MAGURRAN AE (1988) Ecological Diversity and its Measurement. Croom Helm, London.

McCOY JW (1980) The Microbiology of Cooling Water. Chemical Publishing, New York.

ROBERTSON LR (1994) Prevention of microbial adhesion. TAPPI Proc. 225-232.

SCHNEIDER CA, MO K and LISS SN (1998) Applying phenotypic fingerprinting in the management of wastewater treatment systems. Water Sci. Technol. 37 (4-5) 461-464.

VICTORIOL, GILBRIDE KA, ALLENDG and LISS SN (1996) Phenotypic fingerprinting of microbial communities in wastewater treatment systems. Water Res. 30 (5) 1077-1086.

ZAK JC, WILLIG MR, MOORHEAD DL and WILDMAN HG (1994) Functional diversity of microbial communities: A quantitative approach. Soil Biol. Biochem. 26 (9) 1101-1108. 